\title{
INFORMATION QUANTIFICATION OF EMPIRICAL MODE DECOMPOSITION AND APPLICATIONS TO FIELD POTENTIALS
}

\author{
ZAREEN MEHBOOB* and HUJUN YIN ${ }^{\dagger}$ \\ School of Electrical and Electronic Engineering \\ The University of Manchester, Manchester, M60 1QD, UK \\ *zareen.mehboob@postgrad.manchester.ac.uk \\ †hujun.yin@manchester.ac.uk
}

\begin{abstract}
The empirical mode decomposition (EMD) method can adaptively decompose a non-stationary time series into a number of amplitude or frequency modulated functions known as intrinsic mode functions. This paper combines the EMD method with information analysis and presents a framework of information-preserving EMD. The enhanced EMD method has been exploited in the analysis of neural recordings. It decomposes a signal and extracts only the most informative oscillations contained in the non-stationary signal. Information analysis has shown that the extracted components retain the information content of the signal. More importantly, a limited number of components reveal the main oscillations presented in the signal and their instantaneous frequencies, which are not often obvious from the original signal. This information-coupled EMD method has been tested on several field potential datasets for the analysis of stimulus coding in visual cortex, from single and multiple channels, and for finding information connectivity among channels. The results demonstrate the usefulness of the method in extracting relevant responses from the recorded signals. An investigation is also conducted on utilizing the Hilbert phase for cases where phase information can further improve information analysis and stimulus discrimination. The components of the proposed method have been integrated into a toolbox and the initial implementation is also described.
\end{abstract}

Keywords: Neuroinformatics; non-stationary signal processing; empirical mode decomposition; information analysis; mutual information; local field potentials.

\section{Introduction}

Neuronal recordings from electrodes placed in a neural site typically contain activities of both action potentials of neurons as well as extracellular field potentials. ${ }^{1}$ The latter is usually called the local field potentials (LFPs), which represent cortical/ intercortical processing and interneurons activity. This activity can be synaptic potentials, afterpotentials or voltage-gated membrane potentials. ${ }^{1}$ LFPs are low frequency signals as compared to action potentials or spike trains and thus can be separated from the recordings by using a low-pass filter with cutoff frequency of around $300 \mathrm{~Hz}$. The stimulus coding analysis on these two sets of signals also differs. Spike trains are often analyzed on spike rate or spike timing whereas LFPs are analyzed by their spectral properties (magnitude and phase). ${ }^{2-4}$ Rhythmic activations of synapses, which give rise to synchronized oscillations, are reflected in neural recordings such as LFPs (as well as EEG (electroencephalographs)). Such coherent oscillations are a key mechanism of neuronal communication or coding. ${ }^{5,6}$ LFPs have been studied in various neuronal regions such as visual, somatosensory and motor cortex $\operatorname{areas}^{2,7,8}$ for understanding the network behaviors of neurons in these regions. ${ }^{8}{ }^{8}$ Phase relationships also play a vital role in characterizing synchrony among neurons. ${ }^{4,10,11}$ Phase synchronization is a process by which two or more neurons or neuronal networks tend to oscillate with relative phase angles. This effect has been studied in various brain regions. ${ }^{4,12}$ LFPs are also sometimes called deep EEG. EEG is 
noninvasive recordings from electrodes placed on the scalp whereas LFPs are collected from electrodes or microelectrode arrays from deep regions of the brain.

Spectrotemporal analysis such as spectrogram is perhaps the most common approach for neural recordings such as LFPs and offers many advantages. ${ }^{13}$ However, traditional spectral analysis methods, such as Fourier and wavelet transforms, rely on the assumption that the signal is stationary. This is often not the case in neural recordings. Empirical mode decomposition (EMD) ${ }^{14}$ provides a means of adaptively decomposing a non-stationary signal into a number of intrinsic mode functions (IMFs), entirely based on maxima and minima in the data. Spectrotemporal analysis can then be performed on these modes to identify the stimulus-related changes in the neural activity. However, a large number of IMFs can make the analysis difficult and not all the IMFs carry stimulus information.

Here we suggest a method based on EMD to filter through and extract only the information preserving oscillatory modes from the LFPs. We found from the spectral analysis of these modes that they vary greatly in information content and not all of them contribute to stimulus coding, determined by the use of information theory. Phase coding analysis of these IMFs also showed that phase information can help understand the synchronization among the neuronal channels and improve the classification of the responses.

The remainder of the paper is organized as follows. The next section summarizes different analytical methods for continuous field potentials and explains significant advantages of the EMD method. Section 3 presents the procedure for extracting information carrying frequency modes from noisy, non-deterministic LFPs. Analytical applications and results based on the proposed methodology are provided in Section 4. Section 5 gives the initial implementation of the proposed method in a MATLAB toolbox, followed by the conclusions.

\section{Analysis of Local Field Potentials}

Continuous neural recordings, such as LFPs and EEGs, are generally analyzed in different sub-bands. These sub-bands are believed to encode certain features of the stimulus. ${ }^{2,15,16}$ Similar to EEG, LFPs are usually filtered or divided into delta (up to $4 \mathrm{~Hz})$, theta $(4-7 \mathrm{~Hz})$, alpha $(8-12)$, beta $(13-30 \mathrm{~Hz})$, gamma $(30-90 \mathrm{~Hz})$ and high gamma $(60-100 \mathrm{~Hz})$ bands. ${ }^{17,18}$ When setting up an experiment for neural code analysis there are various considerations for stimulus presentation. The stimuli can be simple or complex, discrete or continuous. For example, if the signals are to be collected from the visual cortex, the stimuli can be visual patterns such as grating, color or gray scale images, pictures or videos. Interpretation of LFP (EEG or spikes) coding and information analysis depend on the nature and presentation of these stimuli. For EEG, parametric methods (e.g. autoregressive (AR) models) are typically used for spectral analysis. They lack adaptivity and the results depend on choice of correct model orders for accuracy and tracking changing spectra. ${ }^{19,20}$ Other approaches like independent component analysis (ICA) have been used for single channel EEG analysis. ${ }^{21}$ The drawback is that it is based on the assumption that the signal sources are stationary and disjoint in the frequency domain, which may not be the case.

Fourier or wavelet analysis is usually carried out on a sub-band signal of particular interest. Fourier analysis gets the least preference as it gives a global power-spectrum or frequency distribution and assumes that the signal is stationary. The short-time FT (STFT), a windowed FT, does provide a temporal frequency distribution (spectrogram) given that the signal is stationary in a processing time window. However, it has limited time or frequency resolution, ${ }^{22}$ due to the Heisenberg uncertainty principle. Thus one cannot obtain both time-localized and frequency-localized information with good resolution.

Wavelet transform (WT) is also a widely used time-frequency analysis method. It offers variable time resolutions for high and low frequencies. The performance of WT is dependent on the selection of mother wavelet. Each wavelet addresses differently to the time-frequency resolution problem. Examples of wavelets are Gaussian and Morlet. ${ }^{22}$ Although WT and FT have been used for LFP and EEG analysis, ${ }^{14,17,23,25}$ the trade off between time and frequency energy concentrations is unavoidable due to the uncertainty principle. For this reason WT and STFT cannot simultaneously provide both good frequency and time resolution. WT has been used 
mostly as a denoising technique in multiple channel analysis.

EMD, described in Section 2.1, on the other hand is a relatively new technique which is considered as a form of wavelet transform with the advantage of being adaptive and data-driven. ${ }^{26}$ It considers signal oscillations at local (frequency) level and adaptively decomposes a non-stationary time series into zeromean amplitude mode (AM)/ frequency mode (FM) components called intrinsic mode functions (IMFs). Each IMF shows a natural oscillation presented in the original signal and often lies in a narrow frequency band, this can reveal a particular feature of the given dataset. ${ }^{14}$ From an IMF, one can extract the time-localized and frequency-localized (or instantaneous frequency) information of the signal by using either multitaper method or Hilbert transform. ${ }^{13,17}$ The advantages of EMD over FT can be illustrated in the following examples. The two signals, shown on the left side of Fig. 1, are formed from the same frequency components with different time occurrences.
The Fourier spectra of both, shown on the right side of Fig. 1, are almost the same and do not provide distinct temporal information. Using EMD, signal 1 can be easily decomposed into its original components, as the resulting IMFs shown in Fig. 2. The EMD of signal 2 is the same as the original signal and the resulting only IMF is shown on the bottom right of (Fig. 2). This is because that signal 2 is already a narrow-band oscillation at any particular time. A detailed discussion about significance of EMD for analysis of neural signals can also be found in Refs. 17 and 24.

Thus using EMD as an analytical tool for neural signals gives the advantage that the signals need not to be explicitly band-pass filtered and the EMD adaptively generates the underlying oscillations, each lying in a certain frequency band. These advantages make the EMD method particularly appealing for analysing LFPs as to provide underlying oscillatory responses given a stimulus. An IMF is an oscillation, derived from the original signal that satisfies

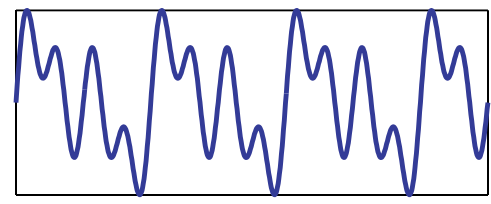

Signal 1

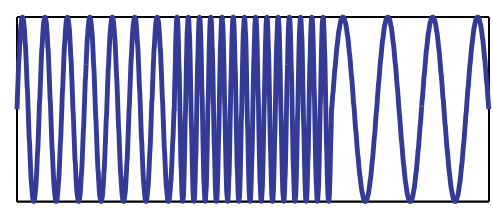

Signal 2

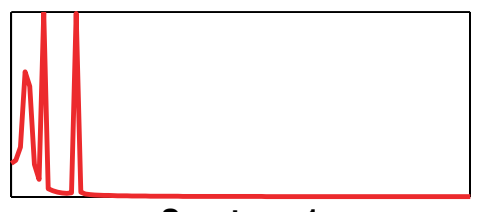

Spectrum 1

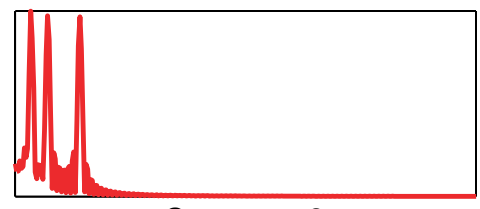

Spectrum 2

Fig. 1. Two multicomponent signals (left) and their spectra (right).
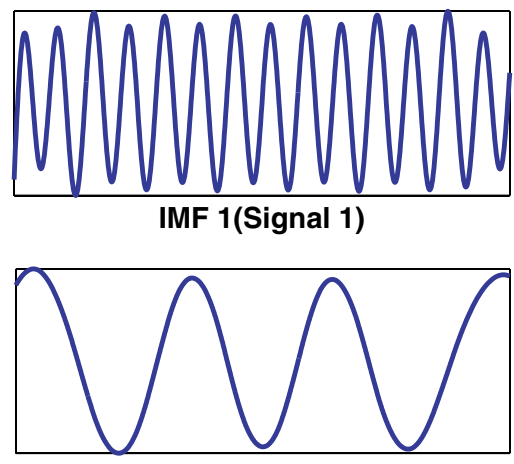

IMF 3 (Signal 1)

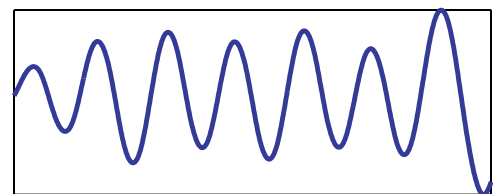

IMF 2 (Signal 1)

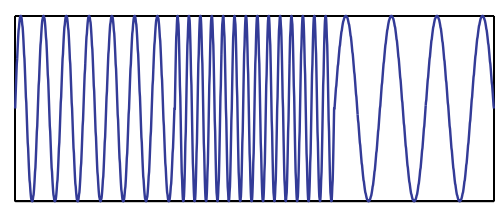

IMF (Signal 2)

Fig. 2. The resulting components (IMFs) of EMD on the signals of Fig. 1. 
two conditions:

- the number of extrema and the number of zerocrossings differs at most by one.

- the local mean is zero.

The EMD method can be carried out online or offline. $^{27}$ In this study, we have used offline EMD in a context that can give insight about the stimulus coding analysis and can be extended to population analysis and information flow analysis within the neuronal group. It has been found earlier, that beta band is active in stimulus coding of motor cortex ${ }^{8}$ and gamma band is informative in visual cortex. ${ }^{15,16}$ EMD has also shown good results in clustering analysis of LFPs. ${ }^{28}$ Recently, EMD has also been combined with ICA for single channel EEG recordings in which ICA is used to extract statistically independent sources after the application of EMD. ${ }^{29}$

\subsection{Empirical mode decomposition}

Given a time series $x(t)$, the EMD is conducted by a sifting procedure given in Table 1. Combining all the IMFs gives the original signal,

$$
x(t)=\sum_{j=1}^{N} I M F_{j}(t)+r_{N}(t)
$$

where $N$ is the total number of IMFs obtained from the time series and $r_{N}(t)$ is the residual. The number of IMFs depends on the nature and length of the recording.

This sifting process is stopped by a criterion based on variance difference between new and previous IMFs. One method is to choose a stop-limit computed from two consecutive sifting results (Eq. (2)).

\section{Table 1. EMD Algorithm.}

1. Identify all the local extrema; then connect all the local maxima by a (cubic) spline to form the upper envelope.

2. Repeat the procedure for the local minima to produce the lower envelope. The upper and lower envelopes covers all the data between them.

3. Find the local mean envelope $m(t)$ by averaging the two envelopes.

4. Extract $m(t)$ from $x(t)$ and obtain $h(t)=m(t)-x(t)$.

5. Check if $h(t)$ satisfies the condition of an IMF. Otherwise repeat the above steps with the residual.
The stop-limit is normally set to $0.2-0.3$. The magnitude of this limit may affect the results and if too small may result in over decomposition of the signal.

$$
\sum_{t}\left(\frac{I M F_{i}(t)-I M F_{i-1}(t)}{I M F_{i-1}(t)}\right)^{2}<\text { Stop limit }
$$

Several EMD algorithms are available on the web. ${ }^{27,30-32}$ We tested these on different time series (data not shown here) and found that the best results were obtained by the implementation provided by Ref. 27. It introduces two thresholds for stopping the sifting process based on both the amplitude of mode and the mean envelope (Eq. (3)). The sifting is iterated until the evaluation function $\sigma<$ Threshold 1 for some prescribed fraction $(1-$ Tolerance) of the total duration, while $\sigma<$ Threshold2 for the remaining fraction. Threshold1 and tolerance are usually set to 0.05 and Threshold2 to 0.5 .

$$
\sigma=\left|\frac{\text { mean }_{\text {amplitude }}}{\text { envelope }_{\text {amplitude }}}\right|
$$

where

$$
\text { mean }_{\text {amplitude }}=\frac{\mid \text { envelope }_{\max }+\text { envelope }_{\min } \mid}{2}
$$

and

$$
\text { envelope }_{\text {amplitude }}=\frac{\mid \text { envelope }_{\max }-\text { envelope }_{\min } \mid}{2}
$$

A typical LFP and its first four IMFs are shown in the left panel of Fig. 3. The right panel shows their spectra, respectively.

\section{Entropy and Information Analysis on IMFs}

This section shows that using the informationcoupled EMD method as a filtering tool can produce a simpler and clearer presentation of nondeterministic LFPs. An initial study has been reported by the authors. ${ }^{33}$ It also gives insight about the information carrying frequencies in the LFP. A block diagram of this framework is shown in Fig. 4.

For information extraction and reduction of uncertainty about a stochastic and nonlinear signal (LFPs, in this case), information measures, entropy (denoted by $H$ ) and mutual information (MI), denoted by $I$, are used. These measures help understanding of neural data by quantifying the probabilities of stimuli and responses. ${ }^{18,34-36}$ Although computationally more intensive, the MI measure is 

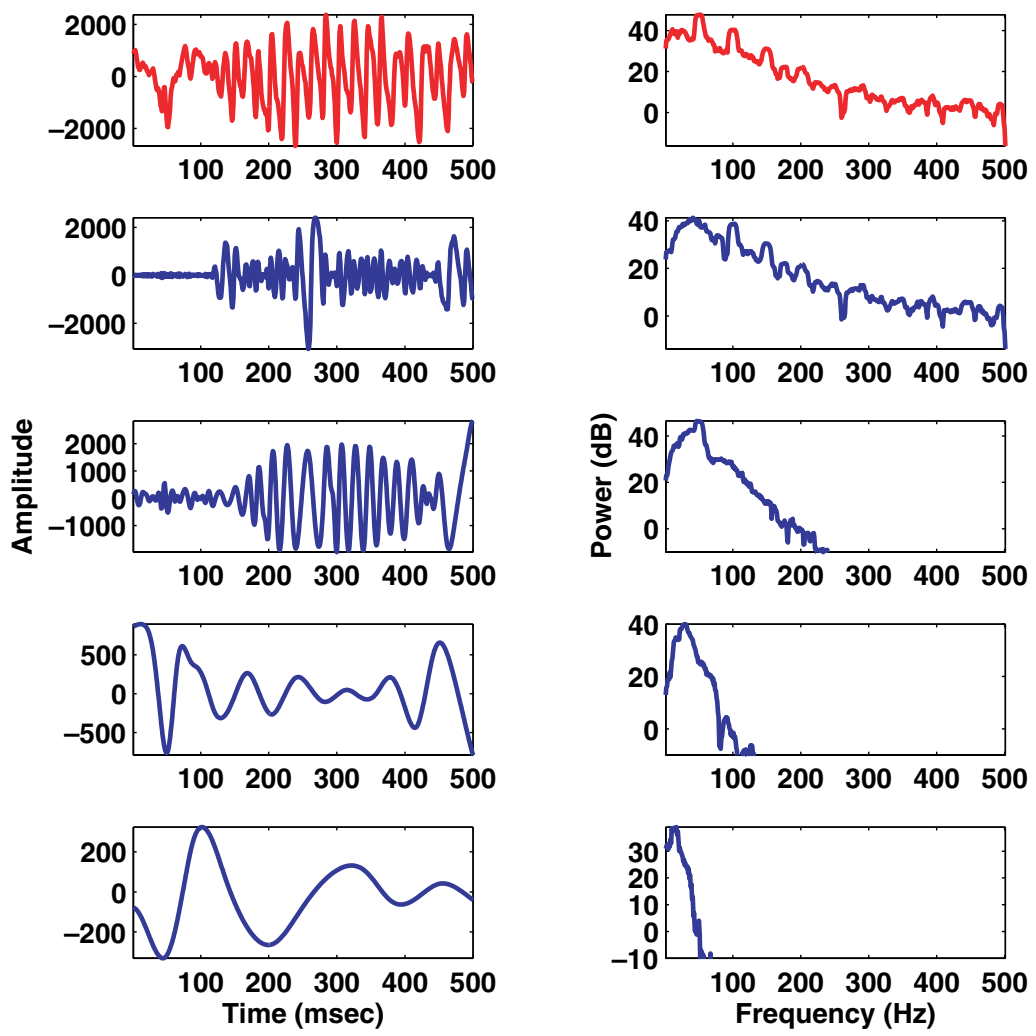

Fig. 3. An LFP recording (top left) and the first four IMFs (left panel), and their spectra (right panel).

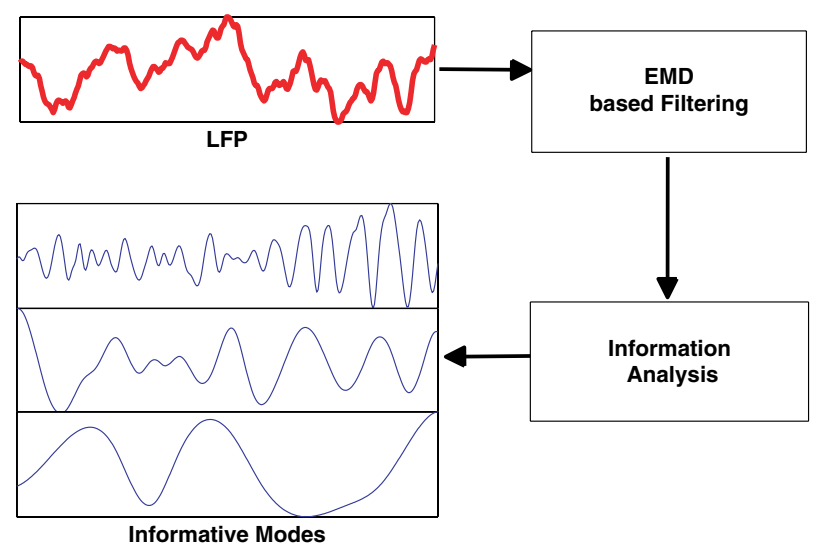

Fig. 4. Block diagram for extraction of informative modes from LFPs.

advantageous over linear measures such as correlation.

The entropy of a response is defined as

$$
H(R)=-\sum_{r} P(r) \log _{2} P(r)
$$

where $P(r)$ is the probability of observing the response.

The conditional entropy, $H(R \mid S)$, is the occurrence of a response $r$ given a particular stimulus $s$ from the stimulus set. ${ }^{18,36}$

$$
H(R \mid S)=-\sum_{s} P(s) \sum_{r} P(r \mid s) \log _{2} P(r \mid s)
$$

The MI between a stimulus and a response, $I(S ; R)$, can be computed from entropy and conditional entropy as,

$$
\begin{gathered}
I(S ; R)=H(R)-H(R \mid S) \\
I(S ; R)=\sum_{s} P(s) \sum_{r} P(r \mid s) \log _{2} \frac{P(r \mid s)}{P(r)}
\end{gathered}
$$

The use of $\log _{2}$ in these definitions means that the MI is in bits and represents the number of bits required to encode the information about the response or stimulus. By observing a response, the uncertainty about the stimulus is reduced by a factor of $2 .^{18,36}$

In case of LFPs, or any continuous signal, the signal is not discrete and can take up to a range of values. In these cases, when calculating the information 
from the spectra of LFP, ${ }^{18}$ one has to discretize the spectral space into bins and then calculate the probabilities of them. This process is often known as binning. The number of bins is often set by the user but it should be less than the total number of unique values in a given series. The number does not change the profile (shape) of information but affects the magnitude slightly. A spectra is often discretized using equally spaced bins. The information equation for LFP takes the form:

$$
I(S ; R)=\sum_{s} P(s) \sum_{r_{f}} P\left(r_{f} \mid s\right) \log _{2} \frac{P\left(r_{f} \mid s\right)}{P\left(r_{f}\right)}
$$

where $r_{f}$ is magnitude power at frequency, $f$.

\subsection{Extraction of information preserving IMFs}

From the spectral information analysis of IMFs from a given recording, it has been found that the information content of each IMF varies, as shown in Fig. 5. From the figure, it is clear that IMFs have different information content per frequency as compared to the original recordings. However, it is not known which other IMFs are taking part in the stimulus coding. For this reason we developed an algorithm
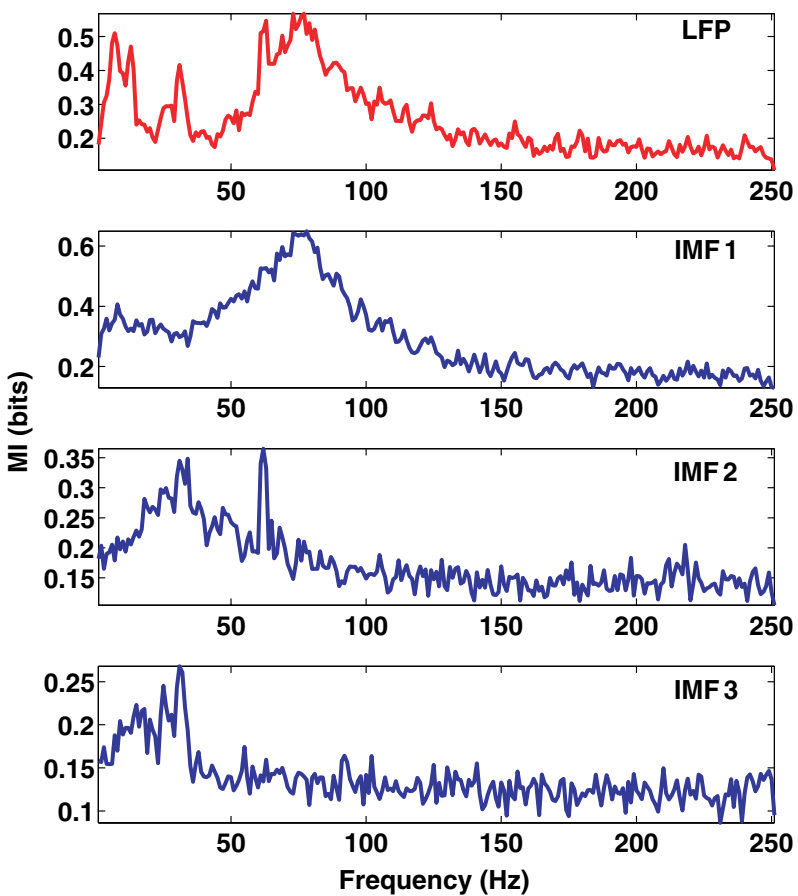

Fig. 5. MI of an LFP recording and information contained in its first three IMFs.
Table 2. Average information content in LFP and corresponding IMFs.

\begin{tabular}{cccccc}
\hline LFP & IMF1 & IMF2 & IMF3 & IMF4 & IMF5 \\
\hline 0.1183 & 0.0985 & 0.0267 & 0.0108 & 0.0038 & 0.0022 \\
0.2158 & 0.1910 & 0.0398 & 0.0116 & 0.0040 & 0.0023 \\
0.2711 & 0.2481 & 0.0457 & 0.0174 & 0.0116 & 0.0071 \\
0.1804 & 0.1477 & 0.0331 & 0.0152 & 0.0051 & 0.0045 \\
0.1449 & 0.1275 & 0.0335 & 0.0129 & 0.0047 & 0.0034 \\
0.1311 & 0.1191 & 0.0296 & 0.0092 & 0.0034 & 0.0019 \\
0.2150 & 0.1789 & 0.0307 & 0.0156 & 0.0084 & 0.0066 \\
\hline
\end{tabular}

that can be used to extract the information carrying modes from LFP recordings. The proposed method computes information content from the spectral attributes of decomposed IMFs. In this way it also quantifies the spectral coding in LFPs.

Table 2 shows the average information content of several LFP recordings and their IMFs. The data is from a set of channel recordings against visual stimuli. Since the activity is largely in gamma band, which is mainly constituted by the first IMF, the information content in the first IMFs is much greater than other IMFs. For other sensory stimuli, such as auditory stimuli, a lower order IMF may be the dominant information carrier.

The proposed method can be used for data recorded against continuous or discrete stimuli. Discrete stimuli, for example, can be gratings of variable temporal, spatial frequency and orientations. Temporal variations means the speed by which the gratings are presented to the subject and spatial attributes refers to the diameter of gratings. The continuous stimuli are usually visual patterns such as images and videos.

The window size for continuous stimuli recordings depends on the nature of sensory stimuli. The maximum length of the window size is the user's choice or can be selected on basis of particular events, during experiment, at certain time points. However, the minimum length of the window should be equivalent to the time required by the subject to perceive and respond to the given stimulus. For instance, in case of continuous visual stimuli, the window size can be as small as $200 \mathrm{msec}$, as this amount of time is required by a human subject to comprehend the information in a gist of scene. ${ }^{37}$

For discrete stimuli, the data is usually stored in a 4-dimensional array, which takes the form of 
$M \times T \times S \times C$, where $M$ is the number of recordings, $T$ the number of trials, $S$ the dimension of stimuli and $C$ the number of channels. For LFP data against continuous stimuli, dataset usually has 3 dimensions. We denote the response space for continuous stimuli recordings by $R$ and data can be arranged as $R \times T \times$ $C$. For analysis of this case, one can first divide the responses into small stimulus windows (rearrange $R$, $R=M \times S$ ) and then apply the EMD method to them. The other approach is to compute the IMFs first from the whole response space $R$ and then divide the IMFs into stimulus windows. It was found that the latter approach gives better results in terms of frequency resolution and information analysis.

The algorithm for finding the informative IMFs is presented in Table 3. It has been tested on data recorded against both continuous and discrete stimuli. For discrete stimuli, the response matrices do not require any windowing.

Table 3. Algorithm for extracting information preserving IMFs.

1. Apply EMD to each LFP of all trials. This gives $N$ IMFs. $N$ is the number of IMFs obtained in a single trial.

2. Divide each IMF into suitable stimulus windows for continuous stimulus case. For discrete case go to step 4.

3. Calculate power spectrum density of all IMFs using multi-taper method. ${ }^{38}$

4. Divide original LFP into stimulus windows for continuous stimulus case. For discrete case go to step 6.

5. Calculate MI for the LFP using Eq. (8), store it as $I_{L F P}$.

6. Calculate MI for all IMFs using Eq. (8). This gives $I_{I M F}(n), n=1,2, \ldots, N$.

7. Take each $I_{I M F}(n=1, \ldots, N)$ and compare its percentage MI correlation with $I_{L F P}$.

8. Choose the best informative IMF that has the maximum MI correlation with the LFP and store it to a set of $I_{\text {bestIMFs }}$.

9. Choose the next informative IMF by adding each of the remaining $I_{I M F s}$ to the MI of selected IMFs and compute the correlation between their MI and $I_{L F P}$. If the correlation is greater than the previous value +0.05 , it means that this IMF is adding significant amount of information. Choose this IMF as the next best IMF and update the collection of best IMFs. Otherwise quit.

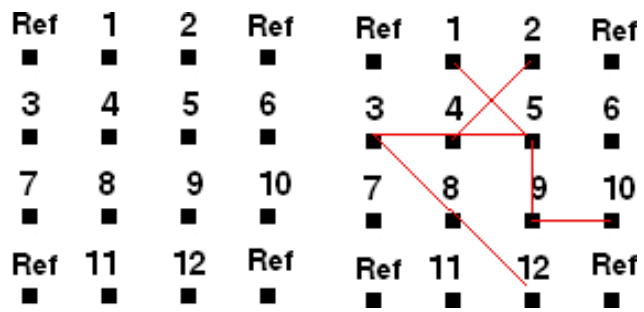

Fig. 6. An example of a $4 \times 4$ microarray of electrodes. The lines on the right side show the information connections between the channels.

LFPs are usually recorded from single electrodes or microelectrode arrays of various sizes, e.g. $4 \times 4$ or $10 \times 10$ arrays. An example is shown in Fig. 6 . Some microelectrodes are used as reference points, connected often onto the brain surface. Data from the rest of the electrodes are then used for analytical purpose. The electrodes are also referred to as channels. The proposed method has been tested on several LFP datasets recorded from multielectrode arrays.

The spectral information for multiple channels can be calculated by Eq. (9). This can be termed as population analysis.

$$
I(C ; R)=\sum_{c} P(c) \sum_{r_{f}} P\left(r_{f} \mid c\right) \log _{2} \frac{P\left(r_{f} \mid c\right)}{P\left(r_{f}\right)}
$$

The information connection quantifies the information coherence between neighboring channels (e.g. $C=2$ ). The results are presented in the next section.

\section{Results and Discussion}

The datasets used in this study were recorded from the visual cortex. These are the LFP recordings against discrete and continuous stimuli represented to the subjects.

\subsection{Single channel analysis}

This section represents analysis and results for single channel LFP recordings against discrete and continuous stimuli.

\subsubsection{Discrete stimuli dataset}

The single channel MI analysis for an LFP dataset against discrete stimuli is presented in Table 4. The first rows represent the most informative IMF and adding more IMFs to them shows combined 
Table 4. Results for single channel analysis against discrete stimuli.

\begin{tabular}{|c|c|c|c|}
\hline IMFs & MI Corr. & IMFs & MI Corr. \\
\hline \multicolumn{2}{|c|}{ Channel 1} & \multicolumn{2}{|c|}{ Channel 2} \\
\hline $\mathrm{IMF}_{1}$ & 0.9218 & $\mathrm{IMF}_{1}$ & 0.8794 \\
\hline $\mathrm{IMF}_{3}$ & 0.9520 & $\mathrm{IMF}_{3}$ & 0.9280 \\
\hline $\mathrm{IMF}_{5}$ & 0.9709 & $\mathrm{IMF}_{5}$ & 0.9654 \\
\hline & - & $\mathrm{IMF}_{7}$ & 0.9708 \\
\hline \multicolumn{2}{|c|}{ Channel 3} & \multicolumn{2}{|c|}{ Channel 4} \\
\hline $\mathrm{IMF}_{1}$ & 0.8872 & $\mathrm{IMF}_{1}$ & 0.7905 \\
\hline $\mathrm{IMF}_{3}$ & 0.9423 & $\mathrm{IMF}_{3}$ & 0.9139 \\
\hline $\mathrm{IMF}_{5}$ & 0.9866 & $\mathrm{IMF}_{5}$ & 0.9811 \\
\hline \multicolumn{2}{|c|}{ Channel 5} & \multicolumn{2}{|c|}{ Channel 6} \\
\hline $\mathrm{IMF}_{1}$ & 0.9050 & $\mathrm{IMF}_{1}$ & 0.7758 \\
\hline $\mathrm{IMF}_{3}$ & 0.9560 & $\mathrm{IMF}_{3}$ & 0.9365 \\
\hline $\mathrm{IMF}_{4}$ & 0.9750 & $\mathrm{IMF}_{5}$ & 0.9770 \\
\hline - & - & $\mathrm{IMF}_{7}$ & 0.981 \\
\hline
\end{tabular}

(increased) information level. For example, only 3 or 4 IMFs out of resultant 8 in total have been found to be the informative ones and adding remaining IMFs has little effect on the overall information level. This is shown in Fig. 7 and Table 4. The first and third columns show the informative IMFs and the second and fourth columns show the cumulative MI correlation values.

Adding insignificant IMFs does not alter the information content, these IMFs can thus be omitted. Examples of information comparison between several LFPs and their information preserving IMFs are shown in Fig. 8. This indicates that few informative IMFs are sufficient, in information sense, in representing and decoding the signal. Note, for an artificially generated LFP or EEG, the profile of the

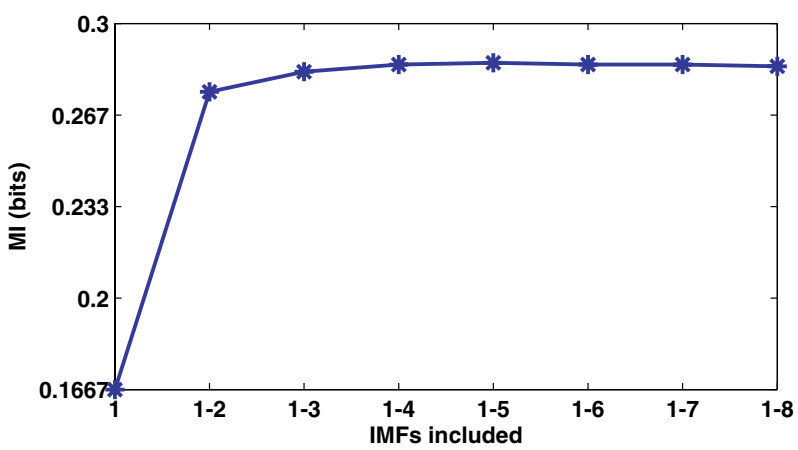

Fig. 7. Cumulative MI levels of dominate IMFs. First 3 IMFs contain most significant information and adding more IMFs has little effect on information.
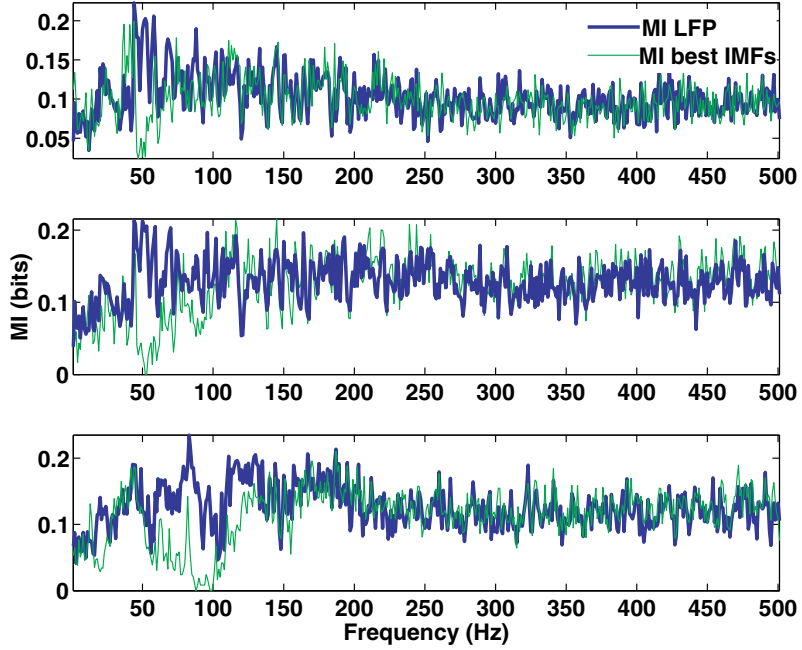

Fig. 8. Information comparison between LFPs and the bestIMFs (information preserving IMFs).

plot would be similar. But for a random dataset it will be simply flat as there will not be any consistent probability distributions and mutual information in random signal.

\subsubsection{Continuous stimuli dataset}

For this dataset, it is found that, the most informative IMFs (1st or 1 st $+3 \mathrm{rd}$ ) which lie in the gamma band are carrying majority of the information. The remaining IMFs have minor effect on the information level. The MI analysis results from four channels are shown in the Fig. 9.

From the figure, it is evident that the peaks of information are in the frequency range between $50-$ $100 \mathrm{~Hz}$. It is also clear that the first IMF is carrying more than $80 \%$ of the information. An LFP recording and the information carrying oscillation $\left(\mathrm{IMF}_{1}\right)$ for the top right case is shown in Fig. 10, clearly showing about $70 \mathrm{~Hz}$ oscillation as the main contribution, which is not directly visible in the original LFP. The EMD has a clear advantage in this regard as these informative oscillations cannot be extracted by other methods like FT or WT.

\subsection{Multiple channel analysis}

The population MI analysis for the data (all channels) recorded against a single stimulus is shown in Fig. 11. The result shows that only two IMFs carry 


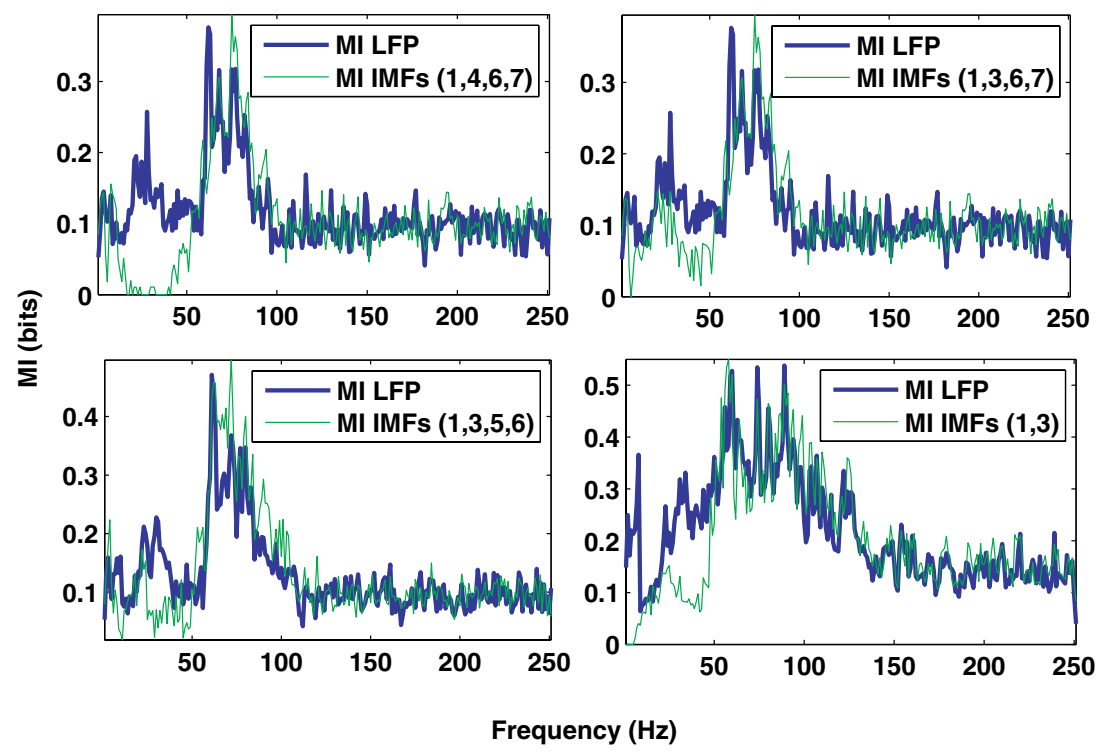

Fig. 9. Results from four channels of the data recorded against continuous visual stimuli.

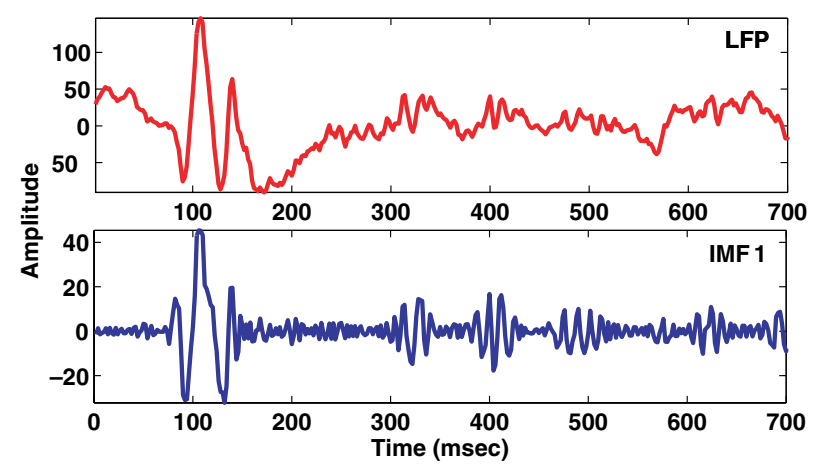

Fig. 10. The LFP recording from the top right channel in Fig. 9 and the extracted information carrying oscillation.

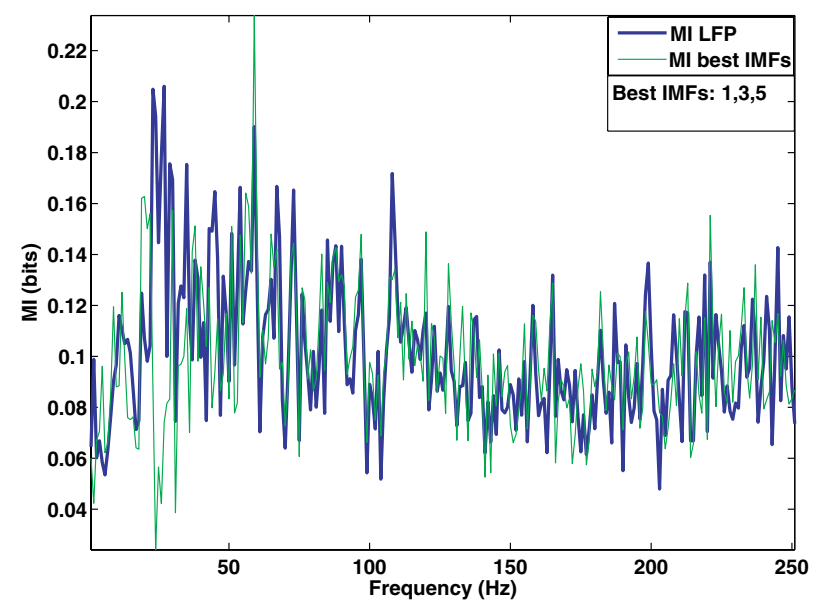

Fig. 11. Population analysis against a discrete stimulus. The responses were collected from a $4 \times 4$ microarray.

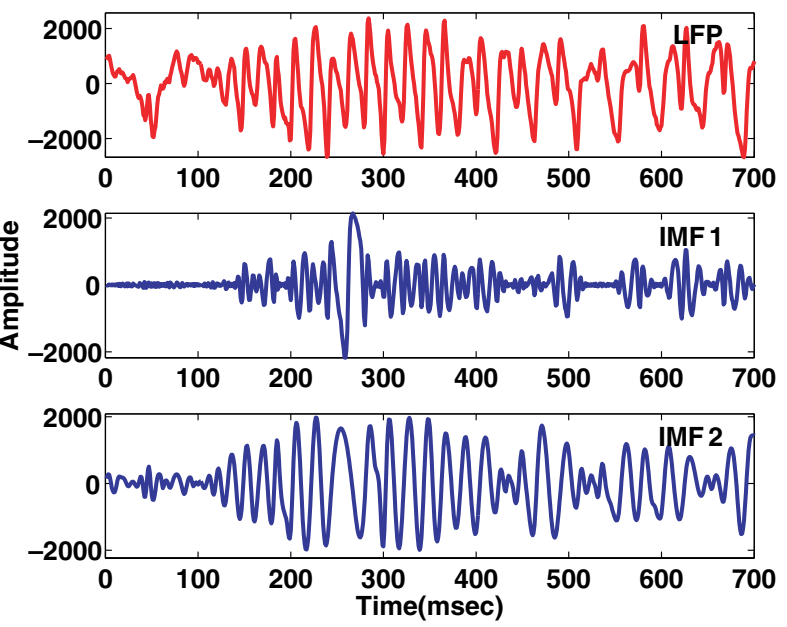

Fig. 12. LFP and the information carrying IMFs for the results shown in Fig. 11.

the most of the information. The extracted information carrying IMFs are shown in Fig. 12. The first IMF (about $90 \mathrm{~Hz}$ ) indicates a high gamma oscillation. The second IMF shows a gamma oscillation (about $50 \mathrm{~Hz}$ ).

\subsection{Information connectivity across channels}

For multichannel recordings, it is possible to study the connectivities based on MI analysis. Connectivity between channels reveals synchronized firing 
patterns among these channels or groups of neurons. Calculation and comparison of MI of LFP recordings from different channels, or their informative IMFs, provide information connectivity between these channels. By introducing delays, one can further analyze causal relationships among different channels. Such analysis help understand information flow or transfer between different brain areas. A typical method for analysing causal relationships between different regions of brain is by means of Granger causality. ${ }^{39}$ We have tested information preserving IMFs to study causal relationships among different channels of EEG recordings. The data used is from an experiment on visual attention of a subject. $9,40,41$ The two stimuli presented are gratings of two different orientations. The subject was rewarded for one stimulus and not for the other. The stimuli are shown in Fig. 13.

The data was recorded from a $10 \times 10$ Utah electrode array and four of the channels were unwired for use as reference points. The proposed method can be applied for information connectivity and synchronization analysis.

The information connectivity diagram for a typical channel is shown in Fig. 14. For all its neighboring channels, the first IMF was found to be the most informative one. The only exception was for channel 18 , for which the information was so low that no IMF
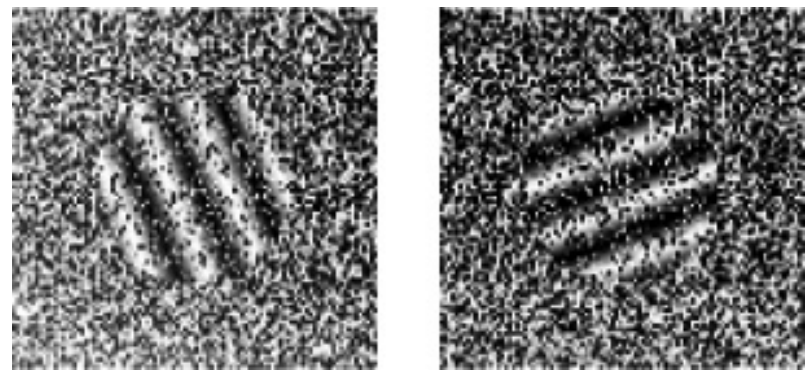

Fig. 13. Rewarded (right) and unrewarded (left) grating stimuli.

was found to be informative with the channel under study. The information profiles also show the levels of information transfer. The dotted lines show weak connectivity while the bold lines show strong connectivity or synchronization between the channels. Alternatively, one can use the thickness of the line to indicate the strength of connectivity.

The analysis has been tested on other channels and Fig. 15 shows the connectivity of most (56 out of 100) channels.

\subsection{Information analysis with Hilbert phase}

The information contained in a continuous signal is confined in its spectral attributes that include both

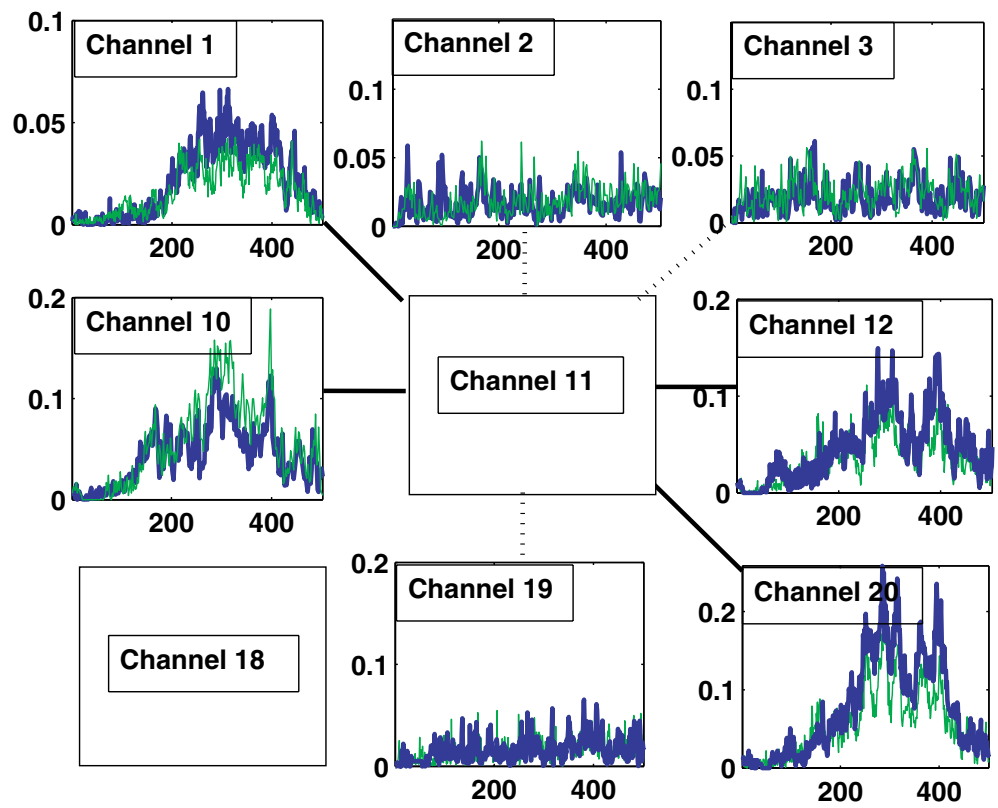

Fig. 14. Information similarity between channel 11 and its neighboring channels. 


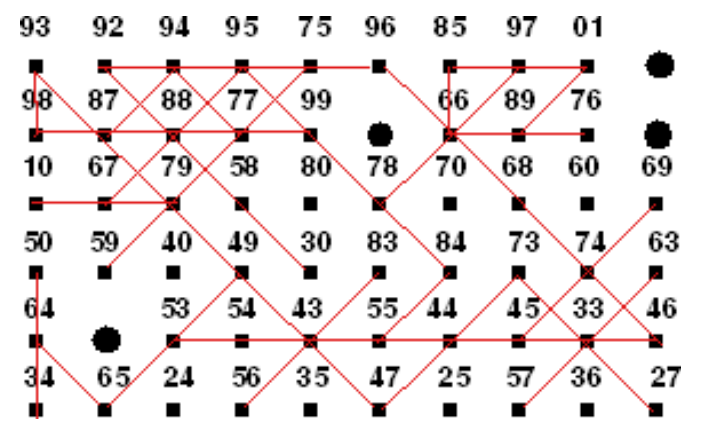

Fig. 15. Information connectivity among channels. The figure only shows the information connections for 56 channels (out of 100 in total).

magnitude and phase. The information extracted from magnitude spectrum may not be sufficient to differentiate certain stimuli. For instance, the information analysis of power spectra (magnitude) for the LFPs with stimuli of Fig. 13 is depicted in Fig. 16. The two stimuli vary on orientation of the gratings. However, from Fig. 16, it is clear that both power spectra lie in the same frequency ranges and have similar profiles. In other words, they are indistinguishable.

For both rewarded and unrewarded stimuli the first IMF was found to be the dominant information carrier. Additional analysis of phase information is carried out. An initial investigation was made in order to compare the information obtained from the Hilbert phase (Eq. 10) of the first IMF of both rewarded and unrewarded stimuli.

$$
\begin{aligned}
I\left(S ; \theta_{I M F}\right)= & \sum_{s} P(s) \sum_{\theta_{i m f}} P\left(\theta_{i m f} \mid s\right) \\
& \times \log _{2} \frac{P\left(\theta_{i m f} \mid s\right)}{P\left(\theta_{i m f}\right)}
\end{aligned}
$$

The Hilbert phase was calculated from the resulting IMFs (A brief introduction of Hilbert transform is given in the appendix). The results of three typical channels are shown in Fig. 17. As can be seen, the information of the phase spectra of the dominating IMFs differs markedly in these two stimuli.

Although Hilbert phase has been used in LFP and EEG analysis, most work has been on analysis of phase synchronization and phase locking phenomena in different frequency bands. Synchronization analysis in EEG can help in distinguishing health issues. ${ }^{43,44}$ One of the recently proposed and tested approaches makes use of EMD and single trial phase locking. ${ }^{11,45}$ The approach has shown some interesting results on EEG analysis. First, the EMD is carried out on EEG signal and then Hilbert phase is calculated for all the IMFs obtained. For each IMF, the series of instantaneous phases are then obtained and phase locking is obtained between all

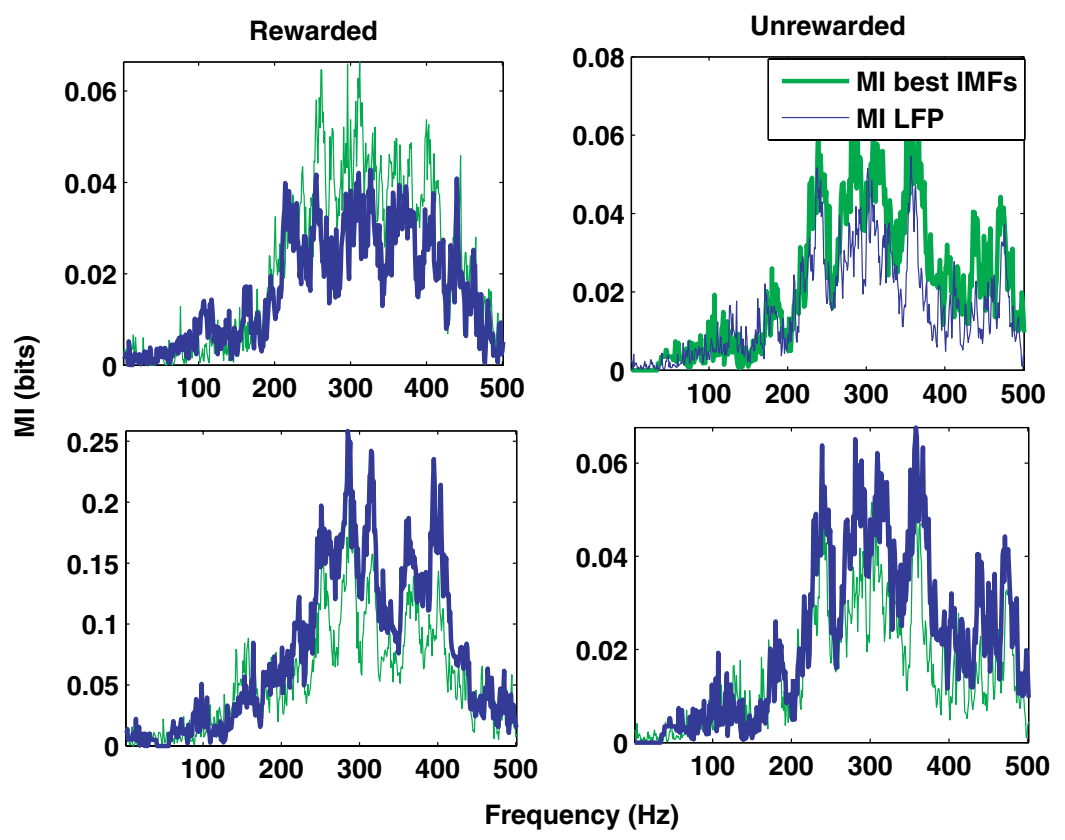

Fig. 16. Indistinguishable information of the two channels for the rewarded and unrewarded stimuli. 

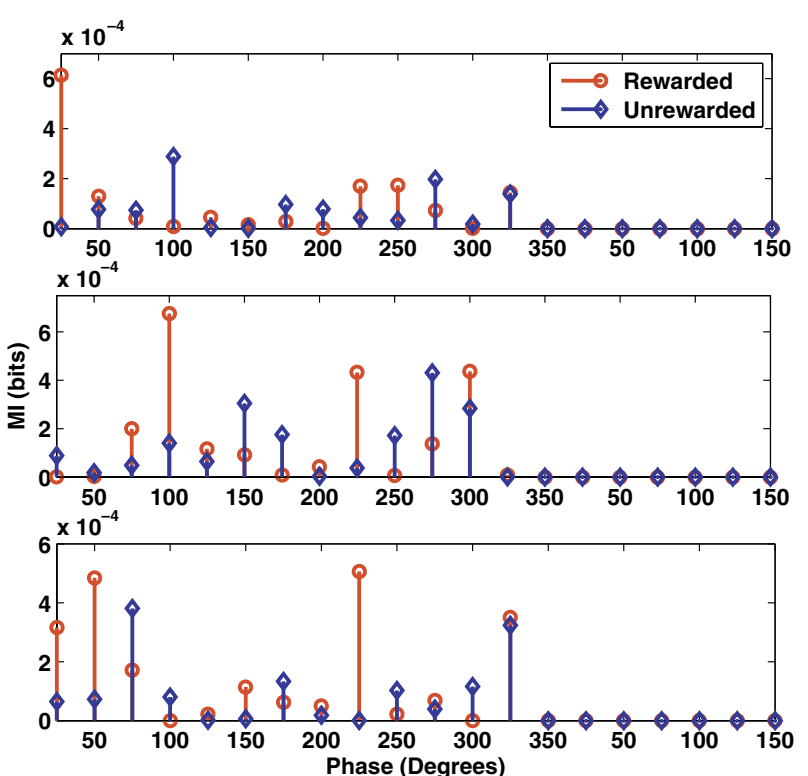

Fig. 17. Information calculated from the phase of the first, dominant IMF of both rewarded and unrewarded stimuli. Stems with circle and diamonds show the information for rewarded and unrewarded stimuli, respectively.

the different IMF combinations by using single-trial phase locking values.

\section{Toolbox Design and Implementation}

The proposed framework has been developed into a MATLAB toolbox for wider generic applications. This section describes the initial design and implementation of this toolbox. The final product is available as a freeware. ${ }^{42}$ The flowchart for the toolbox is presented in Fig. 18.

\subsection{Data input}

The toolbox takes a data file in MATLAB format and assumes that data has already been arranged in $M T S C$ format described in Sec. 3.1. If this is not the case, user can enter the correct dimensions and then toolbox computes information based on the dimensions and parameters given for analysis. When a data file is loaded, its size and dimension information is displayed and then user can enter the parameters for analysis.

\subsection{Input parameters}

Data dimension parameter can take a value of 1-4 depending on the format of data file. The default

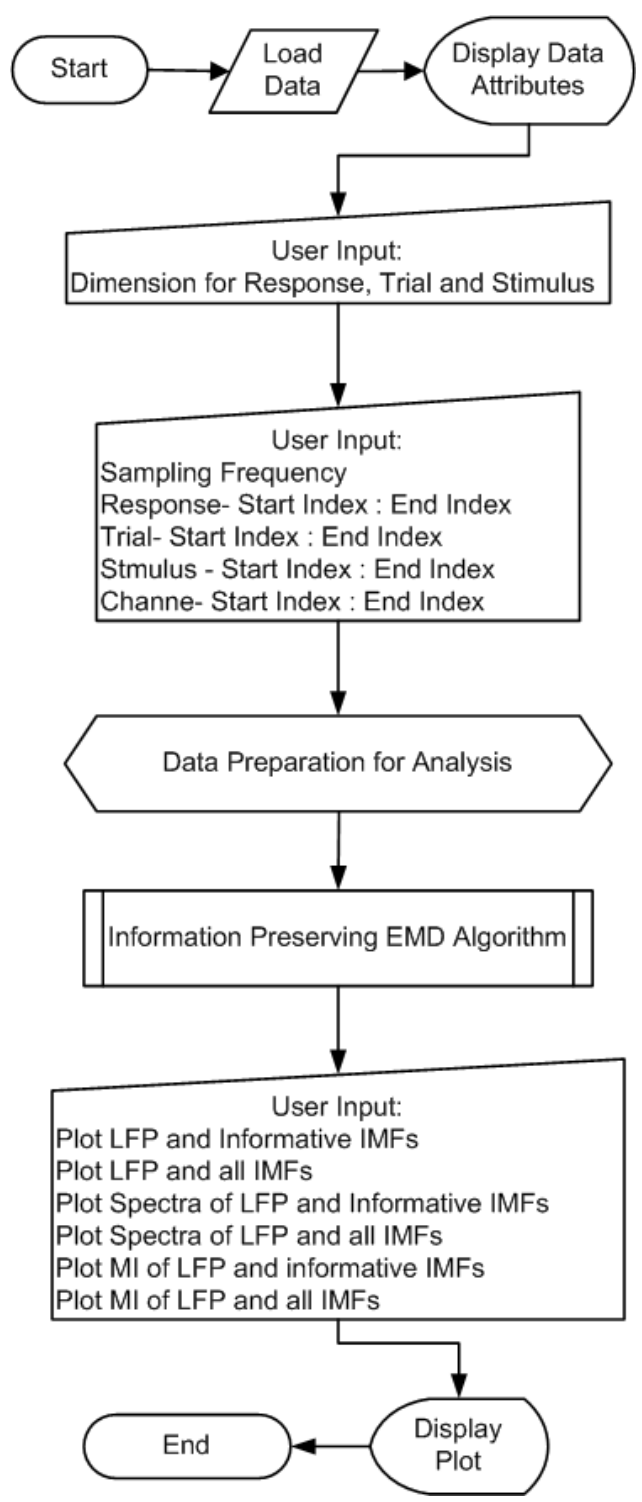

Fig. 18. Flowchart of the toolbox implementation of the method.

sampling frequency is set to $500 \mathrm{~Hz}$ and this is an important parameter since the spectra are calculated based on it and results can be misinterpreted because of it. $1 \mathrm{kHz}$ sampling frequency gives spectra in a range up to $500 \mathrm{~Hz}$ and $500 \mathrm{~Hz}$ sampling frequency gives spectra up to $250 \mathrm{~Hz}$.

\subsection{Toolbox output}

After the completion of analysis, the output window displays an LFP trial at user's choice, its corresponding IMFs and the corresponding information carrying IMFs. User can also view the power 


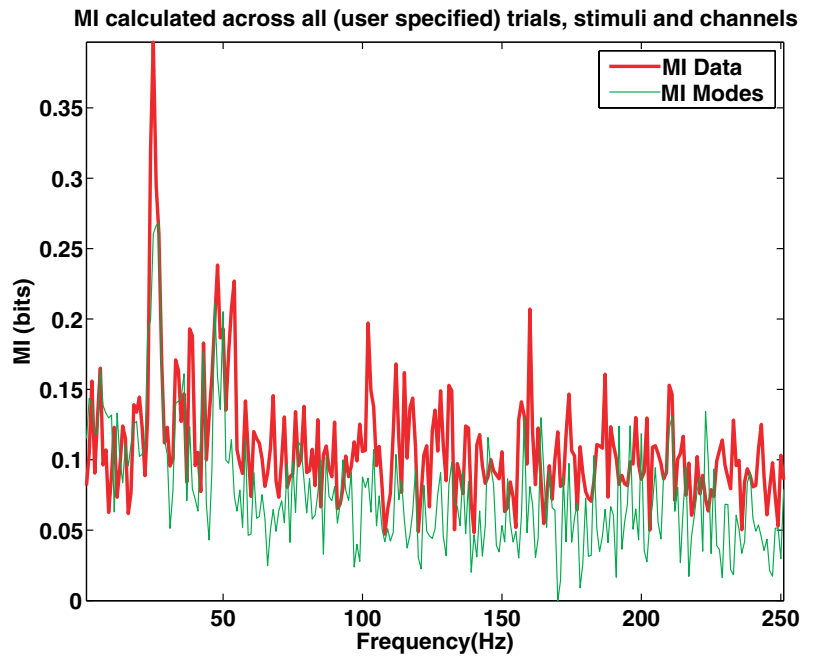

Fig. 19. Toolbox output showing MI of the data and filtered modes.

spectra of the LFP and IMFs. The MI of the original recording and the MI from the informative IMFs are also displayed. A snapshot from an LFP result is shown in Fig. 19.

\section{Conclusion}

An information-coupled EMD framework has been developed for analysis of local field potentials and EEGs. EMD decomposes a non-stationary, nonlinear signal into a number of intrinsic oscillations; while information preserving EMD extracts few (1 to 3 usually) most informative IMFs only. These information carrying IMFs reveal the dominating oscillations in the signal, thus can greatly simplify the analysis of potentially complex signals and facilitate their interpretation. The proposed method has been applied to LFPs from single and multiple channels and the information analysis has shown the information carrying frequency bands and the underlying oscillations that otherwise are not directly visible in the signals. The method can also be applied for information connection analysis among recording channels. The Hilbert analysis of informative IMFs can further help identify discriminating features against different stimuli, when the magnitude information is not sufficient. Further research will incorporate time delay in mutual information analysis in order to reveal information flow (directions) among channels. An initial toolbox has been built to offer the analysis for wider applications.

\section{Acknowledgments}

The authors are thankful for the datasets, helpful comments and guidance provided by R. Vogels, ${ }^{41}$ M.M. Van Hulle and N.V. Manyakov. Z. Mehboob has been funded by the HEC (Pakistan). The authors are also grateful to anonymous reviewers for their helpful comments and suggestions.

\section{References}

1. M. J. Rasch, A. Gretton, Y. Murayama, W. Maass and N. K. Logothetis, Inferring spike trains from local field potentials, J. Neurophys. 99 (2008) 14611476.

2. G. Kreiman, C. P. Hung, A. Kraskov, R. Q. Quiroga, T. Poggio, J. J. DiCarlo, Object selectivity of local field potentials and spikes in the macaque inferior temporal cortex, Neuron 49 (2006) 433-445.

3. S. Ray, S. S. Hsiao, N. E. Crone, P. J. Franaszczuk and E. Niebur, Effect of stimulus intensity on spikeLFP relationship in secondary somatosensory cortex, J. Neurosci. 28 (2008) 7334-7343.

4. A. Kraskov, R. Q. Quiroga, L. Reddy, I. Fried and C. Koch, Local field potentials and spikes in the human medial temporal lobe are selective to image category, J. Cognitive Neurosci. 19 (2007) 479-492.

5. P. Fries, A mechanism for cognitive dynamics: Neuronal communication through neuronal coherence, Trends in Cognitive Sci. 9 (2005) 474-480.

6. M. Vejmelka, M. Palus and K. Susmakova, Identification of nonlinear oscillaotory activity embedded in broadband neural signals, Int. J. Neural Systems 20 (2010) 117-128.

7. A. D. Legatt, J. Arezzo and H. G. Vaughan, Averaged multiple unit activity as an estimate of phasic changes in local neuronal activity: Effects of volumeconducted potentials, J. Neurosci. Meth. 2 (1980) 203-217.

8. D. Rubino, K. A. Robbins and N. G. Hatsopoulos, Propagating waves mediate information transfer in the motor cortex, Nature Neurosci. 9 (2006) 1549-1557.

9. N. V. Manyakov and M. M. Van Hulle, Discriminating visual stimuli from local field potentials recorded with a multi-electrode array in the monkey's visual cortex, in IEEE Workshop on MLSP (2008) 157-162.

10. K. MacLeod, A. Bcker and G. Laurent, Who reads temporal information contained across synchronized and oscillatory spike trains? Nature 395 (1998) 693-698.

11. C. M. Sweeney-Reed and S. J. Nasuto, A novel approach to the detection of synchronization in EEG based on empirical mode decomposition, J. Comput. Neurosci. 23 (2007) 79-111.

12. M. A. Montemurro, M. J. Rasch, Y. Murayama, N. K. Logothetis and S. Panzeri, Phase-of-firing coding 
of natural visual stimuli in primary visual cortex, Current Bio. 18 (2008) 375-380.

13. J. D. Victor, Approaches to information-theoretic analysis of neural activity, Biol. Theory 1 (2006) 302-316.

14. N. E. Huang, Z. Shen, S. R. Long, M. C. Wu, H. H. Shih, Q. Zheng, N. C. Yen, C.C. Tung and H.H. Liu, The empirical mode decomposition and the Hilbert spectrum for nonlinear and non-stationary time series analysis, Proc. the Royal Society 454 (1998) 903-995.

15. J. A. Henrie and R. Shapley, LFP power spectra in V1 cortex: The graded effect of stimulus contrast, $J$. Neurophys. 94 (2005) 479-490.

16. P. Berens, G. A. Keliris, A. S. Ecker, N. K. Logothetis and A. S. Tolias, Comparing the feature selectivity of the gamma-band of the local field potential and the underlying spiking activity in primate visual cortex, Frontiers in Systems Neurosci. 2 (2008) 199207.

17. H. Liang, S. L. Bressler, E. A. Buffalo, R. Desimone and P. Fries, Empirical mode decomposition of local field potentials from macaque V4 in visual spatial attention, Bio. Cyber. 92 (2005) 380-392.

18. A. Belitski, A. Gretton, C. Magri, Y. Murayama, M. A. Montemurro, N. K. Logothetis and S. Panzeri, Low frequency local field potentials and spikes in primary visual cortex convey independent visual information, J. Neurosci. 28 (2008) 5696-5709.

19. D. J. Krusienski, D. J. McFarland and J. R. Wolpaw, An evaluation of autoregressive spectral estimation model order for brain-computer interface applications, in Proc. 28th IEEE EMBS Annual Int. Conf. (2006) 1323-1326.

20. O. Faust, U. R. Acharya, L. C. Min and Bernhard H. C. Sputh, Automatic identification of epileptic and background EEG signals using frequency domain parameters, Int. J. Neural Systems 20 (2010) 159-176.

21. M. E. Davies and C. J. James, Source separation using single channel ICA, Signal Process. 87 (2007) 1819-1832.

22. P. S. Addison, Wavelet transforms and the ECG: A review, Physiol. Measurement 26 (2005) 155-199.

23. M. LeVan Quyen, J. Foucher, J. P. Lachaux, E. Rodriguez, A. Lutz, J. Martinerie and F.J. Varela, Comparison of Hilbert transform and wavelet methods for the analysis of neuronal synchrony, J. Neurosci. Meth. 111 (2001) 83-98.

24. Z. Mehboob and H. Yin, Analysis of non-stationary neurobiological signals using empirical mode decomposition, in Hybrid Artificial Intelligence Systems, HAIS 2008 (2008) 714-721.

25. H. Adeli, Z. Zhou and N. Dadmehr, Analysis of EEG records in an epileptic patient using wavelet transform, J. Neurosci. Meth. 123 (2003) 69-87.
26. P. Flandrin, G. Rilling and P. Goncalves, Empirical mode decomposition as a filter bank, in IEEE Sig. Proc. Lett. (2004).

27. G. Rilling, P. Flandrin and P. Gonalves, On empirical mode decomposition and its algorithms, in IEEEEURASIP Workshop on NSIP, url: http://perso. ens-lyon.fr/patrick.flandrin/NSIP03.pdf (2003).

28. Z. Wang, A. Maier, N. K. Logothetis and H. Liang, Single-trial classification of bistable perception by integrating empirical mode decomposition, clustering, and support vector machine, EURASIP J. on Advances in Signal Process. (2008) 1-8.

29. B. Mijovic, M. De Vos, I. Gligorijevic, J. Taelman and S. Van Huffel, Source separation from singlechannel recordings by combining empirical mode decomposition and independent component analysis, IEEE Trans. Bio. Eng. 57 (2010) 1-9.

30. M. Lambert, A. Engroff, M. Dyer and B. Byer, EMD, url: http://www.owlnet.rice.edu/elec301/Proj ects02/empirical Mode.

31. D. Kim and H. S. Oh, EMD: Empirical mode decomposition and Hilbert spectral analysis, url: http:// cran.r-project.org/web/packages/EMD/index.html (2008).

32. M. Ortigueira, Empirical mode decomposition, url: http://www.mathworks.com/matlabcentral/fileexchange/21409-empirical-mode-decomposition (2008).

33. Z. Mehboob and H. Yin, Information preserving empirical mode decomposition for filtering field potentials, in Int. Conf. IDEAL (2009) 226-233.

34. T. M. Cover and J. A. Thomas, Elements of Information Theory, Wiley Interscience (1999).

35. L. F. Abbot and P. Dayan, Theoretical Neuroscience: Computational and Mathematical Modeling of Neural Systems MIT Press (2001).

36. E. Arabzadeh, S. Panzeri and M. E. Diamond, Whisker vibration information carried by rat barrel cortex neurons, J. Neurosci. 24 (2004) 6011-6020.

37. A. Oliva, Gist of the scene, in Neurobiology of Attention L. Itti and G.Rees and J.K. Tsotsos (eds.) San Diego, CA: Elsevier (2005) 251-256.

38. D. B. Percival and A. T. Walden, Spectral analysis for physical applications: Multitaper and conventional univariate techniques, Cambridge Uni. Press (1993).

39. X. Wang, Y. Chen, S. Bressler and M. Ding, Evaluating causal relations among multiple neurobiological time series: Blockwise versus pairwise Granger causality, Int. J. Neural Systems 17(2) (2007) 71-78.

40. N. V. Manyakov and M. M. Van Hulle, Synchronization in monkey visual cortex analyzed with an information-theoretic measure, Chaos: An Interdisciplinary J. Nonlinear Sci. 18 (2008) 1-7. 
41. E. Franko, A. R. Seiz and R. Vogels, Dissociable neural effects of long term stimulus-reward pairing in macaque visual cortex, J. of Cognitive Neurosc. 22 (2010) 1425-1439.

42. Z. Mehboob, Information preserving EMD based toolbox, url: http://personalpages.manchester.ac. uk/postgrad/Zareen.Mehboob (2010).

43. M. A. Kramer, F. L. Chang, M. E Cohen, D. Hudson and A.J. Szeri, Synchronization measures of the scalp EEG can discriminate healthy from Alzheimers subjects, Int. J. Neural Systems $\mathbf{1 7}$ (2007) 61-69.

44. M. Ahmadlou and H. Adeli, Wavelet synchronization methodology: a new approach for EEG-based diagnosis of ADHD, Clinical EEG Neurosci. 41 (2010) $1-10$.

45. C. M. Sweeney-Reed and S. J. Nasuto, Detection of neural correlates of self-paced motor activity using empirical mode decomposition phase locking analysis, J. Neurosci. Meth. 184 (2009) 54-70.

\section{Appendix}

The Hilbert transform (HT) can be used to obtain the instantaneous attributes of a signal and gives a good time- frequency resolution. Given a signal $x(t)$, its HT is defined as,

$$
y(t)=\frac{1}{\pi} P \int_{-\infty}^{\infty} \frac{x(s)}{t-s} d s
$$

where $P$ is the Cauchy Principle value. The analytic signal is then defined as,

$$
z(t)=x(t)+i y(t)
$$

$x(t)$ is the original signal and $y(t)$ is the HT of it. In terms of phase and amplitude, the analytic signal can be represented as:

$$
\begin{aligned}
& z(t)=a(t) \times \exp (i \theta(t)) ; \\
& a(t)=\sqrt{x(t)^{2}+y(t)^{2}}
\end{aligned}
$$

The phase and instantaneous frequency can be calculated as:

$$
\begin{aligned}
\theta(t) & =\arctan \left(\frac{y(t)}{x(t)}\right) ; \\
f_{\text {inst }} & =\frac{1}{2 \pi} \frac{d(\theta)}{d(t)}
\end{aligned}
$$

